\title{
Time resolved femtosecond spectroscopy of nanocomposites
}

\author{
Oleg Khasanov ${ }^{1, *}$, Tatiana Smirnova ${ }^{2}$, Olga Fedotova $^{1}$, Grigory Rusetsky ${ }^{1}$, Vladimir \\ Gayvoronsky $^{3}$, and Sergey Pokutnyi ${ }^{4}$ \\ ${ }^{1}$ Scientific-Practical Material Research Centre NASB, 19 Brovki, Minsk 220072, Belarus \\ ${ }^{2}$ International Sakharov Environmental University, 23 Dolgobrodskaya, Minsk 220070, Belarus \\ ${ }^{3}$ Physics Institute NASU, 46 Nauky Ave., Kyiv 03680, Ukraine \\ ${ }^{4}$ Chuiko Institute of Surface Chemistry, NASU, 17 Generala Naumova, Kyiv 03164, Ukraine
}

\begin{abstract}
Characterization methods of nanocomposites consisted of semiconductor metal-oxide quantum dots (QD) incorporated into a dielectric matrix have been elaborated on the base of time resolved fourwave mixing and photon echo. Large permanent dipole moment, inherent to QDs under study, local field effect, the QD spatial dispersion and distribution function of the transition dipole moments in QDs are taken into account. New responses at multiple frequencies in directions differed from spatial synchronism conditions of well-known signals have been predicted.
\end{abstract}

As it is well known, nanocomposites attract attention of scientists due to their unique properties. So, nanocrystals $\mathrm{TiO}_{2}$ incorporated into potassium dihydrogen phosphate (KDP) crystals provide enhancement of the second harmonic generation efficiency at higher than $60 \%$ in comparison with pure $\mathrm{KH}_{2} \mathrm{PO}_{4}$. Macroscopic and microscopic features of such nanocomposite is actively discussed but the origin of observed effect is still under question. Bearing in mind high potential for frequency up- and down-conversion of nanocomposites composed of semiconductor metal-oxide quantum dots (QD) incorporated into a dielectric matrix we study exciton states in these materials and elaborate characterization methods accounting for large permanent dipole moment (PDM) of quantum dots (QD).

We dwell on the nanocomposite consisted of semiconductor spherical QD with the permittivity $\varepsilon_{2}$ surrounding insulating matrix with the permittivity $\varepsilon_{1}$, and suppose, that $\varepsilon_{2} \gg \varepsilon_{1}$.In such a system QD-matrix interface forms a potential barrier to electrons not allowing them to penetrate into the QD. The Coulomb interaction of the electron with the QD-matrix interface leads to localization of the electron in the potential well near the QD surface. With increasing QD radius $a$, so that $a>a_{b}$, where $a_{b}$ is the exciton Bohr radius, the spherical QD-matrix interface transforms into a planar interface between the semiconductor and the matrix. The exciton formed from the spatially separated electron and hole becomes two-dimensional (2D) with maximal binding energy. Another dielectric

*Corresponding author: olkhas@mail.ru 
enhancement effect is originated from the field produced by the nanoparticles in matrix, when $\varepsilon_{2} \gg \varepsilon_{1}$ Under these conditions, rich structure of exciton levels is formed with a multitude of possible transitions. It should be noted that in such a nanosystem the PDM and transition dipole moment can exceed the same parameters for volume samples by two orders of magnitude. As a sequence, methods of time resolved laser spectroscopy should be modified accounting PDM impact. Thus, our task reduces to the elaboration of characterization technique of noncentrosymmetric multilevel system by femtosecond pulses. Below we more specifically consider features of time resolved four wave mixing (TRFWM) and photon echo (PE) methods for nanoparticles embedded in noncentrosymmetric matrices with quadratic nonlinearity.

For simplicity, we consider three-level system, which is in resonance with pump pulses at the lowest transition between exciton states. Due to the PDM interaction with the light pulses the Hamiltonian of the light-matter interaction contains fast oscillating diagonal elements that makes impossible traditional theoretical technique application to calculate responses of medium on pulse excitation. After unitary transformation

$$
\hat{L}=\left(\begin{array}{ccc}
\exp (i \lambda) & 0 & 0 \\
0 & \exp (-i \lambda) & 0 \\
0 & 0 & 1
\end{array}\right)
$$

where $\lambda=\frac{p_{11}}{\hbar} \int E_{i} d t=\sum_{m=1}^{\infty} \frac{p_{11} E_{i}}{\hbar \omega} \sin \varphi(z, t), \varphi(z, t)=\left(\omega t-\vec{k}_{i} \vec{r}\right)$, we arrive at Hamiltonian describing QD interaction with a number of fields at multiple frequencies. Here $p_{11}$ is the PDM, $E_{i}, \omega$ and $k_{i}$ are the envelope, carrier frequency and wave vector of $i$-th pulse, correspondingly. Applying successively a number of unitary transformations, we obtain Hamiltonian for generalized two-level system in rotating reference framework. This Hamiltonian does not contain fast oscillating terms and describes one- and two-quantum transitions. While one-quantum transitions occur in the field of excitation pulse $E_{i}$ twoquantum transitions are induced by its components $E_{i} J_{s}(a)$ and $E_{i} J_{s-1}(a)$ at multiple frequencies $(s+1) \omega$ and $s \omega$ due to the PDM contribution. Elaborated approach allows us to deduce the Bloch equations for the QD with PDM in slowly varying envelope approximation and to solve them by means of well-known computational procedures. Besides resonant component macroscopic photoinduced polarization contains multiple harmonics, which can be sources of responses at corresponding frequencies in the nanocomposite. We have calculated TRFWM and PE signals under noncollinear excitation taking into account excitation induced shift of absorption frequency, the QD spatial dispersion and distribution function of the transition dipole moments in QDs. In addition to signals at resonant frequency under well-known spatial synchronism conditions, we predict observation of responses on two-pulse excitation of nanocomposite at multiple frequencies. The observation time and spatial synchronism conditions of new responses will differ from signal in $\left(2 \dot{k_{2}}-\dot{k_{1}}\right)$ direction. The signals at multiple frequencies and terahertz responses are originated from the PDM interaction with excitation pulses. The most intensive signals among all responses will be at double and terahertz frequencies. Signals related to local field effect are discussed. It should be noted that for short time delay between pulses the nonresonant nonlinear matrix will contribute to the TRFWM signals mainly to resonant, second and terahertz harmonics. 